\title{
and continents
}

5 Chenyan $\mathrm{Shi}^{1, \#}$, Lu Zhao ${ }^{2,3, \#}$, Evans Atoni ${ }^{2,3}$, Weifeng Zeng ${ }^{4}$, Xiaomin $\mathrm{Hu}^{5}$, Jelle Matthijnssens ${ }^{1}$,

6 Zhiming Yuan ${ }^{2,3, *}$, Han $\mathrm{Xia}^{2,3, *}$

1. KU Leuven, Department of Microbiology, Immunology and Transplantation, Rega Institute, Laboratory of

Clinical and Epidemiological Virology, Laboratory of Viral Metagenomics, Leuven, Belgium

2. Key Laboratory of Special Pathogens and Biosafety, Wuhan Institute of Virology, Chinese Academy of

Sciences, Wuhan 430071, Hubei, China

3. University of Chinese Academy of Sciences, Beijing 10049, China

4. Liwan Center for Disease Control and Prevention, Guangzhou 510176, Guangdong, China

5. College of Life Science, South-Central University for Nationalities, Wuhan 430074, China

\# These authors contributed equally to this work.

*Correspondence: Zhiming Yuan (Email: yzm@wh.iov.cn) and Han Xia (Email: hanxia@wh.iov.cn) 


\section{Abstract}

33 Mosquitoes belonging to the genus Aedes can efficiently transmit many pathogenic arboviruses, placing a great burden on public health worldwide. In addition, they also carry a number of insect specific viruses (ISVs), and it was recently suggested that some of these ISVs might form a stable species-specific "core virome" in mosquito populations. However, little is known about such a core virome in laboratory colonies and if it is present across different developmental stages. In this study, we compared the viromes in eggs, larvae, pupae and adults of Aedes albopictus mosquitoes collected from the field as well as from a lab colony. The virome in lab-derived Ae. albopictus is very stable across all stages, consistent with a vertical transmission route of these viruses, forming a "vertically transmitted core virome". The different stages of field collected Ae. albopictus mosquitoes also contains this stable vertically transmitted core virome as well as another set of viruses shared by mosquitoes across different stages, which might be an "environment derived core virome". Both these vertically and environmentally transmitted core viromes in Ae. albopictus deserve more attention with respect to their effects on vector competence for important medically relevant arboviruses. To further study this core set of ISVs, we screened 46 publically available SRA viral metagenomic dataset of mosquitoes belonging to the genus Aedes. Some of the identified core ISVs are identified in the majority of SRAs. In addition, a novel virus, Aedes phasmavirus, is found to be distantly related to Yongsan bunyavirus 1 , and the genomes of the core virus Phasi Charoen-like phasivirus is highly prevalent in the majority of the tested samples, with nucleotide identities ranging from $94 \%$ to $99 \%$. Finally, Guadeloupe mosquito virus, and some related viruses formed three separated phylogenetic clades. How

54 these core ISVs influence the biology of mosquito host, arboviruses infection and evolution deserve to be further explored. 


\section{Introduction}

Mosquitoes are highly diverse and widely disseminated. They occupy numerous biotopes and are potential vectors for several pathogenic arboviruses. Specifically, Aedes sp. impose a great threat to global public health. This is due to their ecological plasticity traits that include egg diapause, versatility in using natural and/or urban breeding spots [1] and opportunistic feeding patterns $[2,3]$, which might have promoted their dispersion and adaptation to new uncolonized territories that range from tropical to temperate regions [4].

Moreover, a large number of pathogenic arboviruses, such as Dengue virus (DENV), Yellow fever virus (YFV), Zika virus (ZIKV) and Chikungunya virus (CHIKV), are efficiently transmitted by Aedes (Ae.) mosquitoes, especially Ae. aegypti and Ae. Albopictus [5]. DENV and CHIKV are the most common arboviral diseases, with more than $40 \%$ of the world's population living in areas with transmission of either of these viruses [6, 7]. Particularly, DENV epidemics are becoming a major public health concern in Guangdong Province of China. It experienced an unexpectedly explosive outbreak in 2014 with 45,224 reported dengue fever cases [8], which is more than the total number of cases in the past 30 years in this region [9]. YFV remains endemic in tropical regions in Africa as well as South and Central America, and despite the presence of a good vaccine and large vaccination campaigns in the past, Angola and neighbouring Democratic Republic of the Congo experienced a large outbreak in recent years [10]. ZIKV is the most recent emergent arbovirus, receiving attention worldwide due to its explosive spread in South America, and its association with foetal microcephaly and Guillain-Barré syndrome [11, 12].

Due to the lack of therapeutic treatments and widely available vaccines, control of mosquito population is the most efficient way to prevent arboviral diseases [13]. Mosquitoes are holometabolous insects, whose life cycle goes through four separate stages, including eggs, larva, and pupa in an aquatic habitat and a subaerial adult stage. Although only the females 
81

82

with anthropophilic blood feeding habit can transmit arboviruses, reducing the population of mosquito on the juvenile stages will decrease the number of adults and subsequently lower the transmission chances. The conventional control strategies, including the use of insecticide and environmental sanitation, are facing challenges due to their sustainability and organizational complexity $[13,14]$. In light of the holobiont concept, mosquito-associated microbial communities play an important role in host biology, which may provide new strategies for mosquito control [15].

To date, most of the conducted mosquito microbiota studies have solely focused on the bacterial component in mosquitoes and their possible intrinsic roles in mosquito biology [16]. For example, the nutrient produced by symbiotic bacteria is very important for larvae development [17], and the endosymbiotic alpha-proteobacteria Wolbachia is a natural endosymbiont in several mosquito species and can induce cytoplasmic incompatibility [18]. When this bacterium is introduced into its not-natural host Ae. aegypti, it has strong suppressive effect on DENV infection [19, 20]. More interestingly, some newly discovered mosquito specific viruses have been proposed as future biological control agents against arboviruses [21-23], as novel vaccine platforms [23] or used in diagnostic assays in chimeric virus formation with structural protein genes from arboviruses [23], However, little is known about the virome community dynamics in mosquitoes during its holometabolous development. Some studies have performed viral metagenomics on field mosquitoes collected from different countries (including the United States, Puerto Rico, China, Kenya, Australia, Sweden, etc.), mainly focusing on novel virus discovery [24-32]. In a recent study we showed the presence of a relatively stable "core eukaryotic virome" in field Ae. aegypti and Culex quinquefasciatus mosquitoes from Guadeloupe [33]. However, the presence of insect specific viruses (ISVs) in mosquito lab colonies is barely known, which limit our understanding in their role in the development and physiology of mosquitoes. On the other 
hand, knowing the normal healthy background virome as a reference, will allow us to distinguish between inherent vertically transmitted components and components acquired from the environment of the field mosquitoes, which will benefit the identification of potential viral pathogens and improve the reliability of risk assessment using lab mosquitoes.

110 The aim of this study was to analyze the core virome structure of Ae. albopictus mosquitoes during distinct developmental stages, in a laboratory-derived colony originally obtained from Jiangsu Province, China, and to compare these findings to the virome of field Ae. albopictus mosquitoes collected in Guangzhou (Guangdong Province, China), around 1300 kilometer apart. Furthermore, we conducted a comparative meta-analysis between the viruses identified in this study and 46 publicly available virome SRA datasets of Aedes mosquitoes. Finally, phylogenetic analyses were performed on three selected viruses: Aedes phasmavirus which is highly present in both field and lab Ae. albopictus from China; Guadeloupe mosquito related virus and Phasi Charoen-like phasivirus, which were found in the majority of investigated virome data sets.

\section{Materials and methods}

\subsection{Sample collection}

Field larva, pupa and adult samples of Ae. albopictus were trapped in Guangzhou (Liwan mosquitoes of Ae. aegypti were trapped during July and August of the year 2018 in Ukunda and Kisumu. All samples were collected from residential quarter in urban area, transported to the laboratory using an appropriate cold chain and stored at $-80^{\circ} \mathrm{C}$. Mosquito species were determined by morphological identification and samples were assigned into pools according to date of collection (Table 1). An Ae. albopictus colony was established in our laboratory in 2017 (Wuhan, China), which originated from the stable colony from the 
130 National Institute for Communicable Disease Control and Prevention, China CDC (Beijing,

131 China). The adult mosquitoes were held at $\left(27-30^{\circ} \mathrm{C}\right)$, with $60-85 \%$ relative humidity, and a

132 photoperiod of 12:12 h light-dark cycle. The larvae were fed with a mixture of yeast powder

133 and wheat mill. Adult mosquitoes were placed into cages $(30 \mathrm{~cm} \times 30 \mathrm{~cm} \times 30 \mathrm{~cm})$ and the

134 males were fed with $10 \%$ sucrose solution, while the females were fed with blood from mice.

135 The egg, larva, pupa, male and female adult samples of lab colonies mosquitoes were

136 collected respectively in August of 2017 (Table 1).

Table 1 - Aedes spp. from China and Kenya used for viral metagenomic sequencing

\begin{tabular}{lllllll}
\hline Sample names & Mosquito species & $\begin{array}{l}\text { Collection } \\
\text { years }\end{array}$ & Habitats & Locations & $\begin{array}{l}\text { Mosquito } \\
\text { numbers used } \\
\text { for sequencing }\end{array}$ & $\begin{array}{l}\text { Obtained } \\
\text { trimmed reads } \\
\text { number }\end{array}$ \\
\hline 17-Lab-egg & Ae. albopictus & 2017 & Lab & Wuhan, Hubei, China & 200 & $23,801,510$ \\
17-Lab-larva & Ae. albopictus & 2017 & Lab & Wuhan, Hubei, China & 250 & $22,990,930$ \\
17-Lab-pupa & Ae. albopictus & 2017 & Lab & Wuhan, Hubei, China & 250 & $18,066,298$ \\
17-Lab-adultF & Ae. albopictus & 2017 & Lab & Wuhan, Hubei, China & 250 & $20,109,482$ \\
17-Lab-adultM & Ae. albopictus & 2017 & Lab & Wuhan, Hubei, China & 250 & $19,227,638$ \\
17-GZ-larva & Ae. albopictus & 2017 & Field & Liwan district, Guangzhou, China & 2000 & $29,564,646$ \\
17-GZ-adult & Ae. albopictus & 2017 & Field & Liwan district, Guangzhou, China & 1000 & $37,766,072$ \\
18-GZ-larva & Ae. albopictus & 2018 & Field & Liwan district, Guangzhou, China & 2000 & $35,580,984$ \\
18-GZ-pupa & Ae. albopictus & 2018 & Field & Liwan district, Guangzhou, China & 500 & $25,744,126$ \\
18-GZ-adult & Ae. albopictus & 2018 & Field & Liwan district, Guangzhou, China & 1100 & $29,644,914$ \\
18-Kenya-1 & Ae. aegypti & 2018 & Field & Ukunda, Kwale, Kenya & 1100 & $17,758,834$ \\
18-Kenya-2 & Ae. aegypti & 2018 & Field & Kisumu, Kisumu, Kenya & 840 & $26,678,724$ \\
\hline 138
\end{tabular}

138

$139 \quad 2.2$ Sample preparation for NGS

140 Pooled mosquito samples were triturated by a Tgrinder electric tissue grinder OSE-Y30

141 (TIANGEN,China) on ice using sterile pestles with $200 \mu \mathrm{l}$ of Roswell Park Memorial

142 Institute (RPMI) medium. Mosquito homogenates were clarified by centrifugation at

$14320,000 \times \mathrm{g}\left(4^{\circ} \mathrm{C}\right.$ for $\left.30 \mathrm{~min}\right)$ and filtered through a $0.45 \mu \mathrm{m}$ membrane filter (Millipore,

144 Billerica, USA). Supernatants were collected and stored at $-80^{\circ} \mathrm{C}$. RNA was extracted from

145 the supernatants per the manufacturer's instructions by using RNeasy mini kit (Qiagen,

146 Germany). Then strand-specific libraries were prepared using the TruSeq ${ }^{\circledR}$ Stranded Total 
RNA Sample Preparation kit (Illumina, USA). TruSeq PE (paired-end) Cluster Kit v3 (Illumina, USA) and TruSeq SBS Kit v3 - HS (300 Cycles) (Illumina, USA) were used for sequencing with $150 \mathrm{bp}$ per read (PE $2 \times 150 \mathrm{bp}$ ), which was performed at Shanghai Biotechnology Corporation with the Illumina HiSeq 2500 platform (Illumina, USA).

\subsection{Bioinformatic analysis}

The obtained raw paired-end reads were trimmed for quality and adapters using Trimmomatic [34] and the remaining reads were de novo assembled into contigs using metaSPAdes [35]. Contigs from all pools longer than 500bp were filtered for redundancy at $95 \%$ nucleotide identity over $80 \%$ of the length using ClusterGenomes [36]. The representative contigs collection was classified using DIAMOND [37] against the $\mathrm{nr}$ database (updated on 29th Sep 2019) on sensitive mode for taxonomic annotation. KronaTools [38] were used to parse the output file of DIAMOND, which found the least common ancestor of the best 25 DIAMOND hits (based on BLASTx score) for each contig. All contigs annotated as eukaryotic virus were extracted using an in-house python script. The abundance and length coverage of eukaryotic virus contigs in individual pool were obtained by aligning trimmed and decontaminated reads of each samples to the representative contigs collection using BBMap [39]. Abundance tables from eukaryotic virus were extracted and further used for making heatmaps in $\mathrm{R}$ with the ComplexHeatmap [40], ggplot2 [41], phyloseq [42] packages. The length coverage of each viral species per sample was calculated by dividing the contigs length covered (by at least one read) by the total length of the contigs.

\subsection{Eukaryotic virus screening of Aedes mosquito virome data in SRA}

To investigate the level of conservation of the eukaryotic core viruses identified in Chinese samples from this study with those of other Aedes mosquitoes worldwide, we retrieved 46 published SRA datasets (including 25 datasets of Aedes sp. from Guadeloupe [33], eight from 

and one from China [45]) (Additional file 1 and Fig. 1). Two more viral metagenomic data of Ae. aegypti collected in Kenya were also used (Table 1 and Fig. 1). The world map displaying the geographic origin of all Aedes virome datasets used in this study were drawn with ArcGIS (ArcMap 10.5). The trimmed reads of the two datasets of Ae. aegypti from Kenya were de novo assembled using metaSPAdes [35]. The raw reads of SRA data sets were de novo assembled by SKESA [46] with default settings. These obtained contigs were taxonomically annotated using DIAMOND against the nr database (updated on 29th Sep 2019) on sensitive mode. KronaTools [38] and an in-house python script were used to parse the output file of DIAMOND and extracted eukaryotic virus contigs.

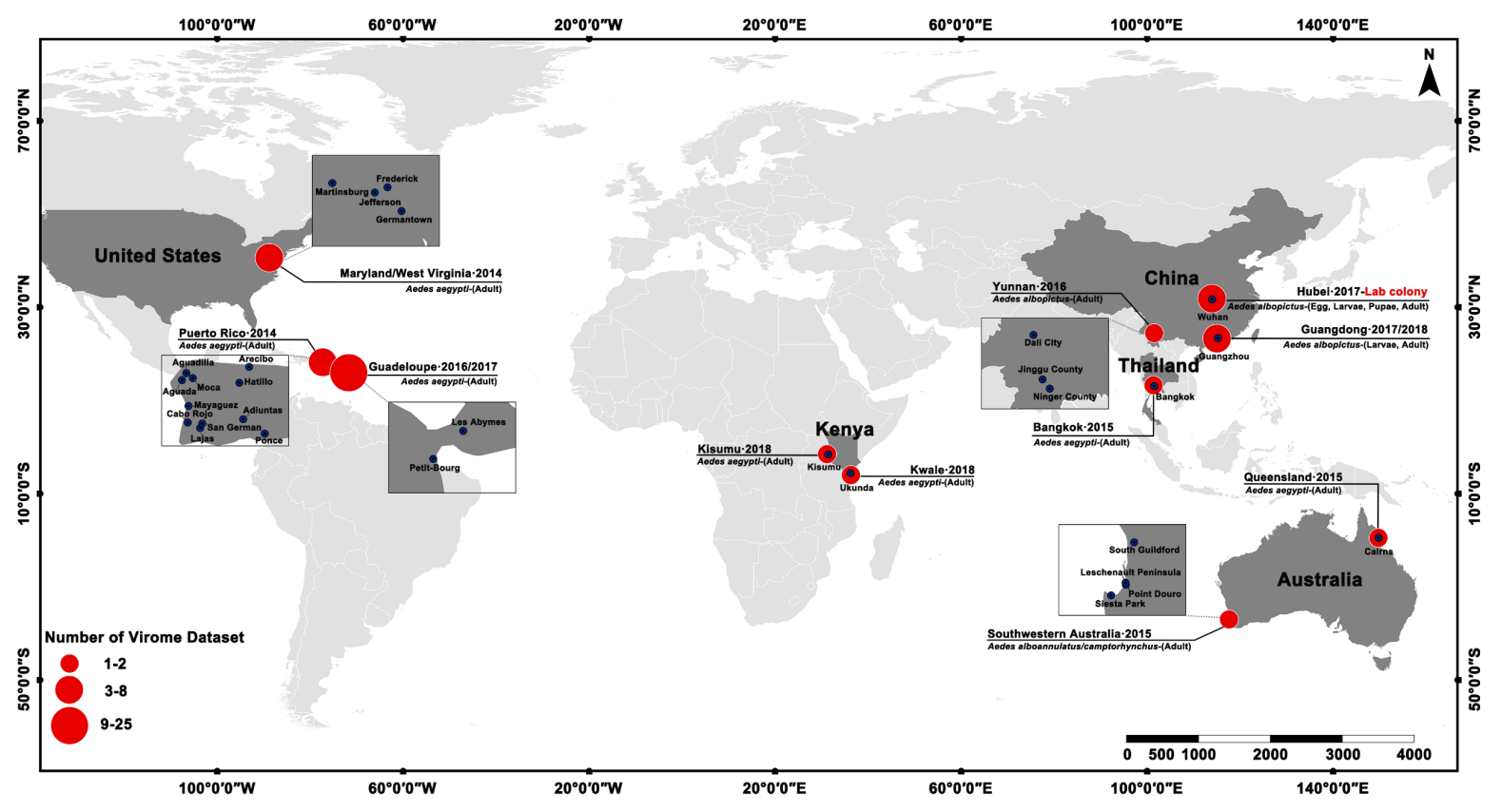

Fig 1. Global distribution of Aedes mosquitoes virome datasets used in this study

\subsection{The presence of eukaryotic virus in all Aedes virome datasets}

Eukaryotic virus contigs longer than $500 \mathrm{bp}$ from all 58 Aedes virome datasets were merged 
species are shown in the heatmap (Figure 3) only if they were present in samples from at least three countries and contain at least one contig that was longer than $1500 \mathrm{bp}$.

\subsection{Phylogenetic analysis}

To obtain the complete genomes related to Yongsan bunyavirus 1 (YBV1), Phasi Charoenlike phasivirus (PCLPV) and Guadeloupe mosquito virus (GMV) from all Aedes virome datasets, the trimmed reads of each sample were individually mapped (with BWA [47]) against the selected reference genomes: 17-Lab-egg-L (MT361040), 17-Lab-egg-M (MT361040) and 17-Lab-pupa-S (MT361044) as the reference for YBV1, PCLPV isolate Rio (KR003786.1, KR003784.1 and KR003785.1) for PCLPV and 18-GZ-larva-seg1 (MT361057) and 18-GZ-pupa-seg2 (MT361060) for GMV. The consensus sequences of these viruses were generated from the bam files using samtools and bcftools [48]. Accession numbers of these viruses obtained from our dataset are in Additional file 2 and viral genome sequences recovered from SRA datasets can be found in

https://github.com/Matthijnssenslab/AedesVirome.

The nucleotide sequence of the complete genome or complete coding region of each genome (segment) of these viruses were used to determine the evolutionary history of the discovered viruses together with appropriate reference strains from GenBank. Alignments of sequences were performed with MAFFT v7.222 [49] using the most accurate algorithm L-INS-I with 1000 cycles of iterative refinement. Ambiguously aligned regions were removed by trimAl v1.2 [50] using automated trimming heuristic, which is optimized for Maximum-Likelihood (ML) phylogenetic tree reconstruction. Phylogenetic trees for each segment were reconstructed from 1,000 ultrafast bootstrap maximum likelihood (ML) tree replicates using 


\section{Results}

212

213

214

215

\subsection{Comparison of the eukaryotic virome in field and lab Ae. albopictus}

The egg, larva, pupa and adult (male and female) pools from a lab colony in Wuhan, as well as the larva, pupa and adult pools from the field in Guangzhou underwent the metagenomic sequencing and an average of 27 million trimmed reads were obtained per pool (Table 1), which were de novo assembled into 1,657,229 contigs in total. The clustering of 71,303 contigs longer than $500 \mathrm{bp}$ from all samples at $95 \%$ nucleotide identity over $80 \%$ of the length results in 56,419 representative contigs. According to BLASTx annotation results using Diamond, the majority of the representative contigs (93\%) belonged to Eukaryota (mainly derived from the mosquito host genome) and 179 contigs were assigned as eukaryotic viruses. Eukaryotic viral reads in each pool occupied $0.2 \%$ to $1.8 \%$ of trimmed reads, except for pool 17-GZ-larva which contained $15.8 \%$ viral reads. Most of the eukaryotic viral contigs were annotated as belonging to 80 different viruses (including several viruses with segmented genomes), although some of these had very low similarities to known viruses in the database (Fig. 2). No known pathogenic arboviruses were identified, and the closest relatives of the identified viruses were generally found in insects or plant.

Only 20 of the 80 viral species belonged to an established viral genus or family (e.g. Flavivirus, Iflavirus, Phasivirus, Quaranjavirus, Rhabdoviridae, Virgaviridae, Totiviridae, Nodaviridae), whereas the remaining ones are currently unclassified.

The heatmap in Fig. 2 displays the mapped read numbers against each of the representative (partial) viral genome as well as the length coverage of assembled contigs of each virus (Additional File 3 and 4). The virome in field Ae. albopictus collected from both 2017 and 2018 showed significantly higher richness and diversity compared to that in lab-derived mosquito pools. The adjusted p-value of Wilcoxon test on Chaol and Shannon index were 0.012 and 0.008 , respectively (Additional File 5). The virome profile of the different pools of 
236 lab colony are relatively stable, except that several viral species are absent or low abundant in

237 the larvae pool (Fig. 2). The wild larvae, pupa and adult pools collected in GZ in 2018 and

238 the adult pool in 2017 also display a very similar viral community. However, the larvae pool

239 collected in GZ 2017 contained more than 30 additional unique viral species with almost

$240100 \%$ length coverage, which are almost completely absent in the adults collected at the same

241 time and in the same place. Furthermore, the lab and field Ae. albopictus have 20 viral

242 species in common. Among them, some contigs only distantly related (47\% BLASTx

243 identity) to YBV1, are present in all lab and field derived mosquito pools with a high

244 abundance and length coverage. This is also true for Phasi Charoen-like phasivirus, and 245 contigs annotated as Yongsan tombus-like virus 1, except in field mosquitoes collected in 246 2018. Some viruses such as Phasi Charoen-like phasivirus appear to be present in in higher

247 levels in the lab-derived Ae. albopictus samples. In contrast, reads of some viruses appear to 248 be present in higher number in the field samples, as is the case for Guato virus and 249 Guadeloupe mosquito mononega-like virus. Furthermore, some viruses are only present in 250 field mosquitoes among all pools with high abundance, such as Hubei partiti-like virus 22, 251 Hubei mosquito virus 2 and Wenzhou sobemo-like virus 4. 


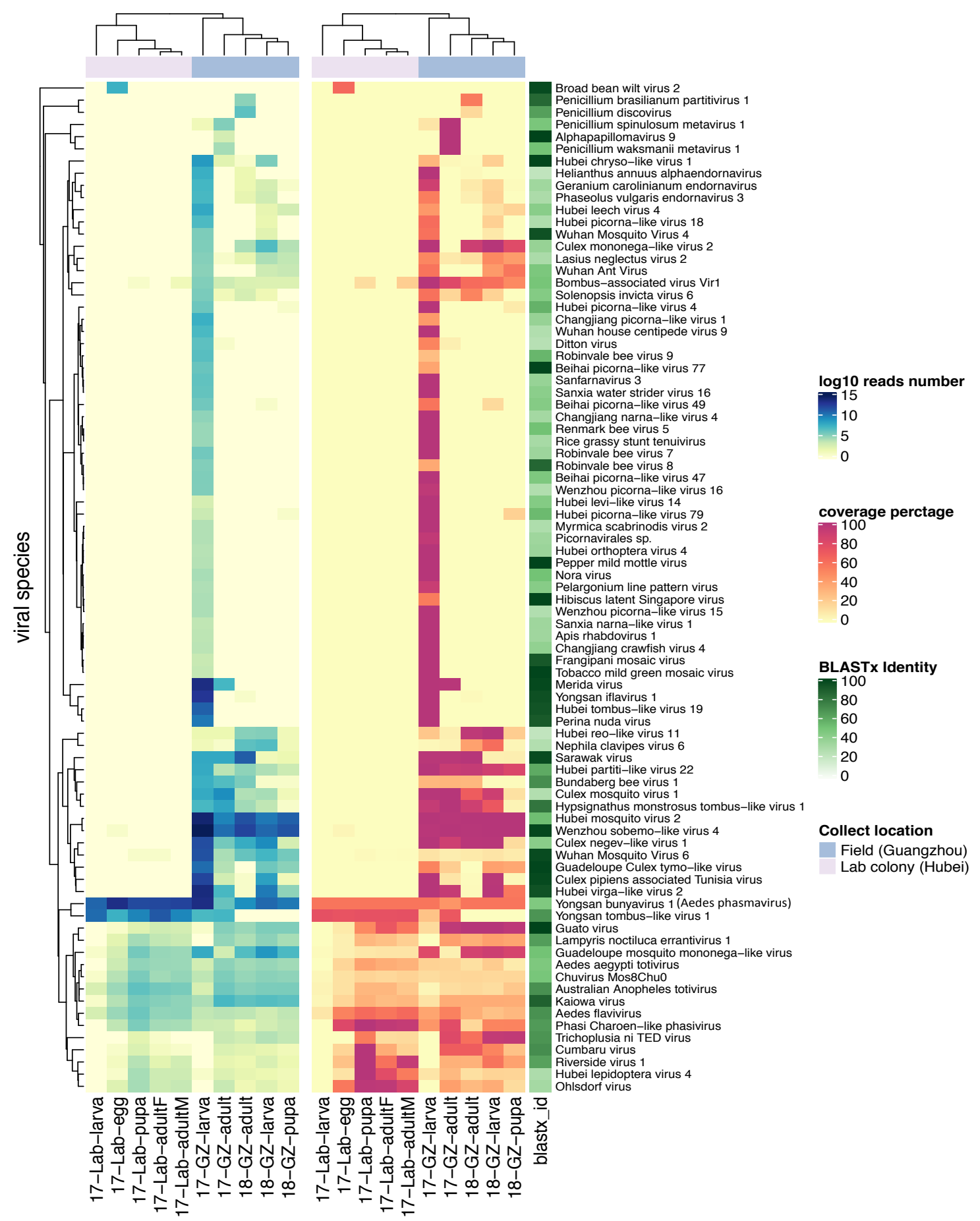
pools. The heatmaps show the total mapped reads number on a log10 scale (left) and length coverage (right) of assembled contigs of each virus. The hierarchical clustering is based on the Bray-Curtis distance matrix calculated from the $\log 10$ reads number. The viral species names shown in the heatmap are from the taxonomic annotation by DIAMOND and KronaTools. For each of the contigs assigned to a particular species, the ORF with the highest BLASTx identity to a reference sequence was taken, and the median identity of these different ORFs is shown in the shaded green bar. 


\subsection{Prevalence of viruses in public SRA data sets derived from Aedes mosquitoes}

All 46 available viral metagenomic data of Aedes sp. from public database (SRA) together with two samples from Kenya (Additional file 1) were further analyzed to determine the prevalence of conserved ISVs in various Aedes mosquitoes. These samples were collected from locations in different continents including the USA, Puerto Rico, Australia, Thailand, Guadeloupe, China and Kenya. The mosquito species in the majority of samples are Ae. aegypti, except the one from China (Ae. Albopictus) and five from Southwestern Australia (Ae. alboannulatus or Ae. Camptorhynchus). The heatmap in Fig. 3 shows the presence and absence of 42 viruses in the 58 analyzed virome data sets, grouped per collection locations and viral families. Only the viral species present in minimum three locations and containing at least one contig longer than $1.5 \mathrm{~kb}$ are displayed in the figure. Notably, the total number of viral species present in samples from the USA and Puerto Rico are much less than in other locations, which may due to the low sequencing depths, , or different viruses present in these samples.

Totiviridae is the most prevalent viral family, containing two highly abundant species (Aedes aegypti totivirus and Australian Anopheles totivirus) with positivity rates of $63.8 \%$ and $60.3 \%$, respectively. Phasi Charoen-like phasivirus in the Phenuiviridae is present in all four Aedes species and all locations (except USA, which only has three viruses presented). In addition, the families Flaviviridae, Orthomyxoviridae, Rhabdoviridae and Xinmoviridae also contain viral species present in 5 or more locations and at least Ae. aegypti and Ae. albopictus mosquitoes. For the unclassified viral species, Chuvirus Mos8Chu0 and Kaiowa virus are present in 40 and 35 out of 58 samples, respectively, and were found in all four Aedes species. Guadeloupe mosquito virus is detected in field Ae. aegypti and Ae. albopictus virus (absent in lab-derived and wild Ae. albopictus from this study) are found in field Ae. 


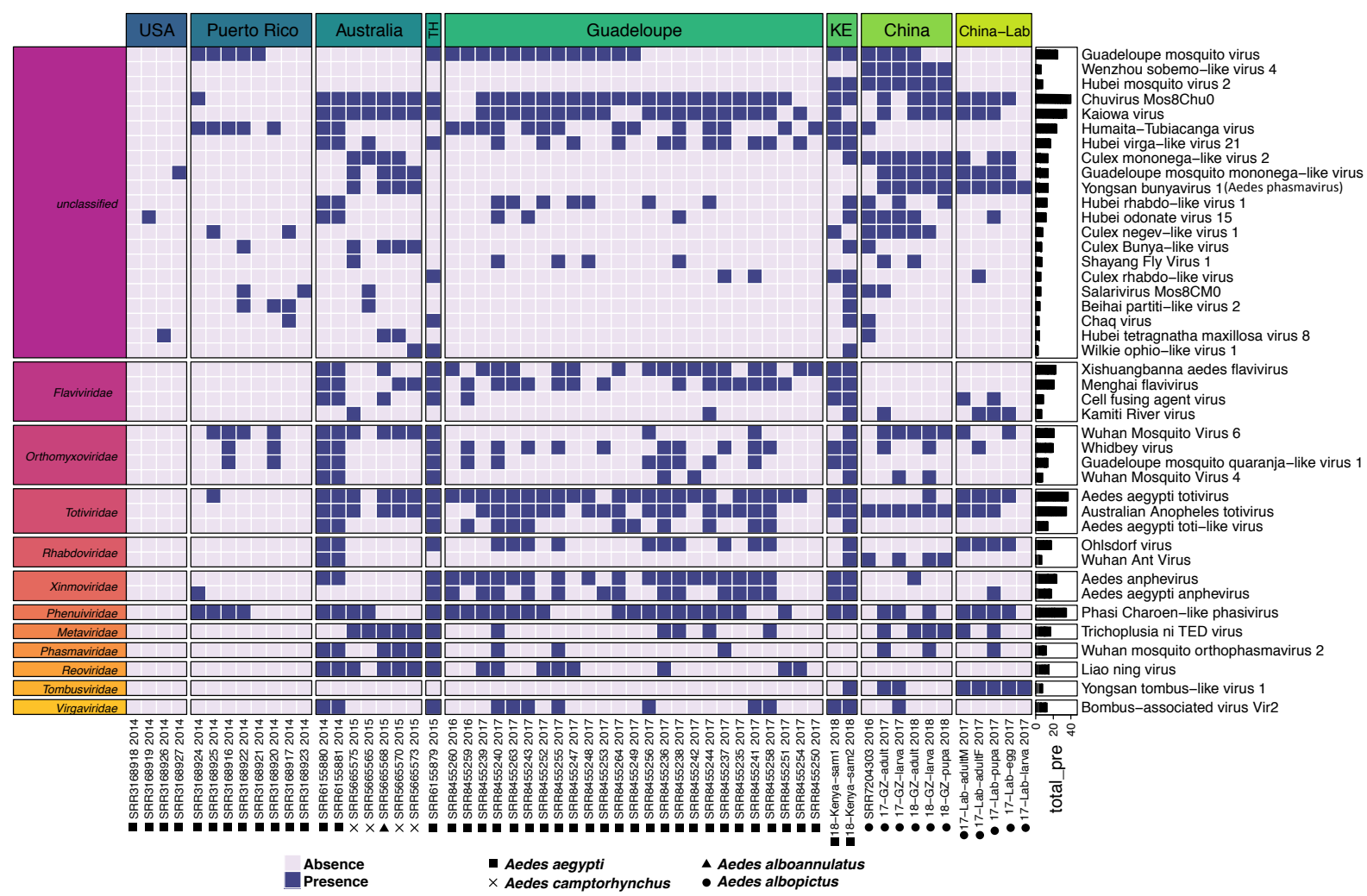

Fig. 3 Presence and Absence of viral species in Aedes sp. virome datasets. The viral species shown in the heatmap present in samples from at least three locations and contain at least one contig that is longer than $1500 \mathrm{bp}$. The specific viral species was considered as presence in the sample, as long as the sample had one contigs (>500 bp) assigned to the species.

\subsection{Phylogeny of three selected viruses}

Phylogenetic analysis was conducted on three selected viruses related to YBV1, which genomes related to YBV1, PCLPV and GMV from analyzed viral metagenomic data of 

virus of this family (tentatively named as Aedes phasmavirus in this study) distantly related to YBV1 (vide infra) was presented in lab Ae. albopictus from China and filed Aedes mosquitoes collected from three countries, including China, Korea, and Australia (Table 2). PCLPV, a widely distributed mosquito viruses, has been identified from lab colonies, mosquito cell lines and wild Aedes mosquitoes in eight counties or regions (China, Puerto that is maintained in nature through vertical transmission [55]. A recent study has reported that GMV is closely related to Wenzhou sobemo-like virus 4 and Hubei mosquito virus 2 [56]. GMV related virus has been detected only in mosquito sample collected from the field, indicating it is likely acquired horizontally from the environment. 
bioRxiv preprint doi: https://doi.org/10.1101/2020.04.23.058701; this version posted April 24, 2020. The copyright holder for this preprint (which was not certified by peer review) is the author/funder. All rights reserved. No reuse allowed without permission.

Table 2 The distribution of three selected viruses

\begin{tabular}{|c|c|c|c|c|c|}
\hline & region & origin & $\begin{array}{l}\text { Aedes } \\
\text { phasmavirus } \\
\text { (relate to YBV1) }\end{array}$ & PCLPV & GMV related virus \\
\hline \multirow[t]{3}{*}{ lab } & China & 2017-Wuhan-lab & + & + & \\
\hline & Belgium & 2018-Belgium-lab & & + & \\
\hline & UK & 2017-Bristol-lab & & + & \\
\hline \multirow[t]{17}{*}{ field } & China & 2016-Zhejiang & & + & + \\
\hline & & 2017-Guangzhou & + & + & + \\
\hline & & 2018-Guanghozu & + & + & + \\
\hline & Puerto Rico & 2014-Puerto Rico & & + & + \\
\hline & Thailand & 2008-Thailand & & + & \\
\hline & & 2015-Thailand & & + & + \\
\hline & Kenya & 2018-Kenya1 & & + & + \\
\hline & & 2018-Kenya2 & & + & + \\
\hline & USA & 2014-USA & & & \\
\hline & & 2015-USA & & + & \\
\hline & Australia & 2014-Australia & & + & \\
\hline & & 2015-Australia & + & + & \\
\hline & & 2016-Australia & & + & \\
\hline & Korea & 2016-Korea & + & & \\
\hline & Guadeloupe & 2016-Guadeloupe & & + & + \\
\hline & & 2017-Guadeloupe & & + & + \\
\hline & Brazil & 2012-Brazil & & + & \\
\hline
\end{tabular}




\subsubsection{Phylogeny of Aedes phasmavirus}

335

336

In both lab and field derived Ae. albopictus sequence data from China in 2017 and 2018, three contigs of Aedes phasmavirus (APV) in each sample were found to share highest amino acid identities of $50.8 \%, 48.1 \%$, and $43.6 \%$ with S-, M- and L-segments of YBV1 (MH703047.1, MH703046.1, MH703045.1), respectively. In total, 10 viral genomes of APV with three segments could been recovered from all lab and field Ae. albopictus pools of this study in China (17-lab-egg, 17-lab-larva, 17-lab-pupa, 17-lab-adultM, 17-lab-adultF, 17-GZlarva, 17-GZ-adult, 18-GZ-larva, 18-GZ-pupa, 18-GZ-adult) with the longest lengths of 1185, 2022, and $6468 \mathrm{nt}$ corresponding for the complete cds (coding sequence) of the nucleocapsid, glycoprotein and RNA-dependent RNA polymerase ( $\mathrm{RdRp})$ genes, respectively. For all three segments, the nucleotide identity among the 10 assembled viral genomes ranged between $96 \%$ and $100 \%$, which indicated they represent closely related variants. In the phylogenetic analysis of the three segments separately, the 10 viral genomes of APV clustered within the genus Orthophasmavirus in the family of Phasmaviridae (Fig. 4). They closest relative was YBV1 identified in Ae. vexans mosquitoes collected in South Korea in 2016, but with low nucleotide similarities (ranging from 52\% to 57\%) in the coding regions of the S-, M-, and Lsegments. Thus, the APVs presented in the lab and field Ae. albopictus in China appears to represent a novel species in the genus Orthophasmavirus. 
bioRxiv preprint doi: https://doi.org/10.1101/2020.04.23.058701; this version posted April 24, 2020. The copyright holder for this preprint (which was not certified by peer review) is the author/funder. All rights reserved. No reuse allowed without permission.
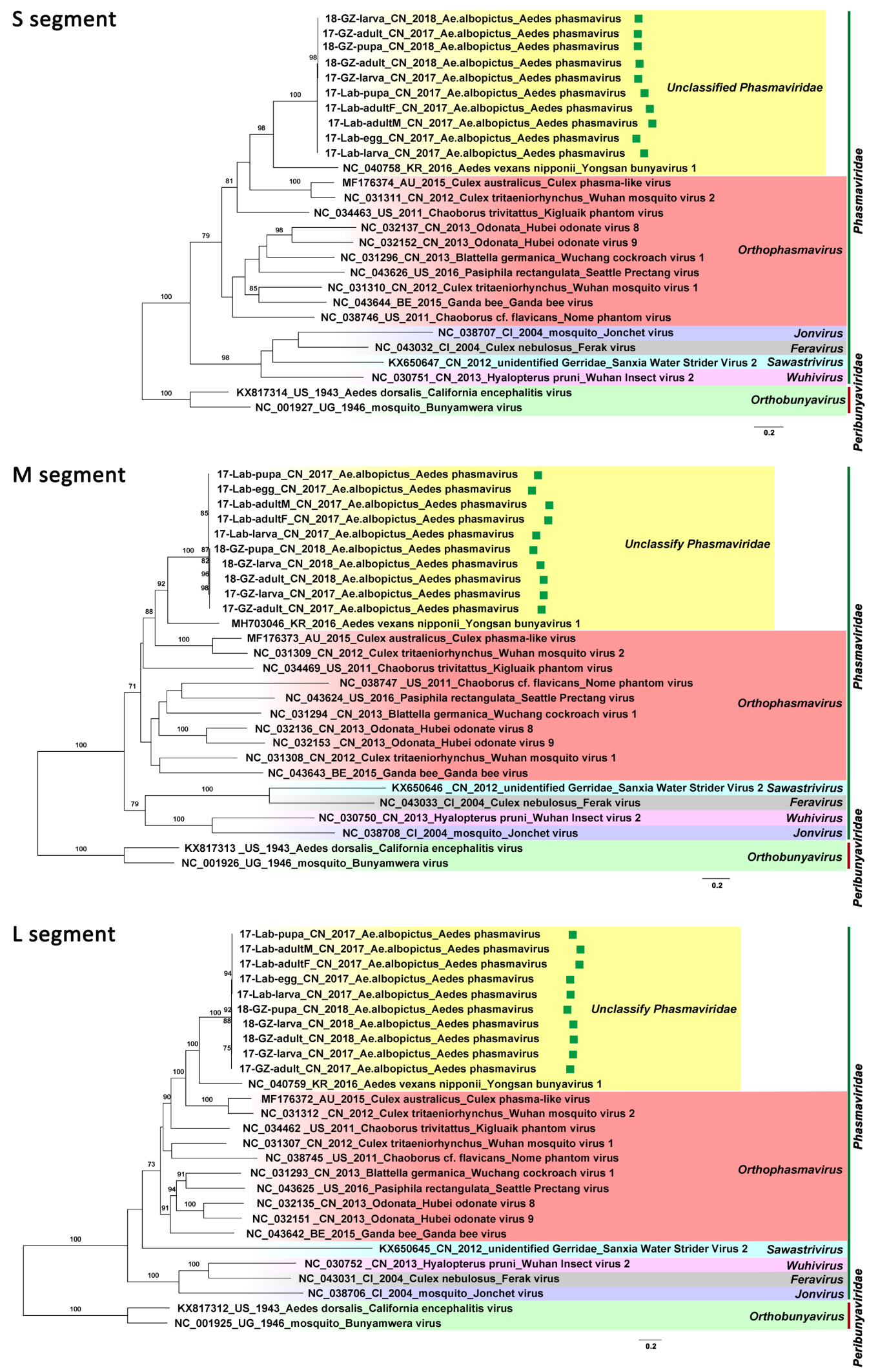

Fig. 4. Maximum likelihood phylogeny on the nucleotide level of complete cds region in $S, M$, and $L$ segment of the newly found Orthophasmaviruses in Aedes mosquitoes from China (highlighted with green square) against other representative members in family Phasmaviriade. Representatives in Orthobunyavirus belonged to the 


\subsubsection{Phylogeny of Phasi Charoen-like phasivirus}

359 Since the abundance of Phasi Charoen-like phasivirus (PCLPV) reads in the Ae. albopictus

360 from Guangzhou and lab-derived are relatively low, no complete genome was recovered from

361 these pools. All three segments of PCLPV with a complete coding region were successfully

362 recovered in one Ae. aegypti sample from Puerto Rico collected in 2014 and two Ae. aegypti samples from Kenya collected in 2018. The phylogenetic analysis was performed on these newly identified PCLPVs, all other PCLPV genomes in GenBank (including the ones identified in Ae. aegypti mosquitoes from Thailand in 2008, from US in 2015, from Australia in 2016, from Zhejiang Province of China in 2016, from Guadeloupe in 2017 and in Aag2 cells from UK) and two more genomes obtained from lab Aag2 cell lines in Belgium (Fig. 5). It is interesting to note that all these PCLPVs originating from distant geographic locations, different years and field mosquitoes vs. lab cell lines, all cluster very closely in the three segments, respectively. 

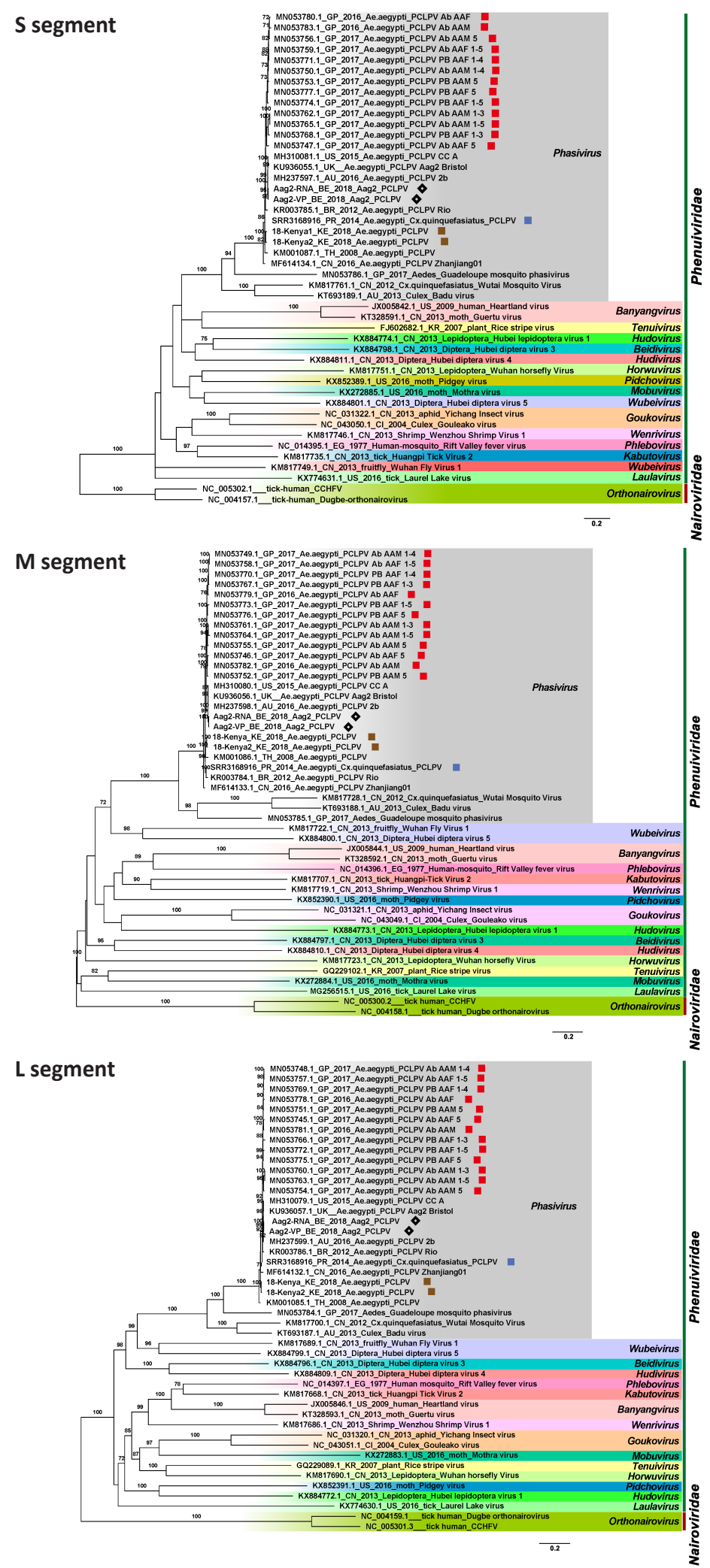

Fig. 5 Maximum-likelihood phylogenetic tree based on nucleotide sequence of complete coding regions of the

$376 \mathrm{~S}, \mathrm{M}$, and $\mathrm{L}$ segment of PCLPV identified from Aedes mosquitoes and cells, together with other representative 377 members of Phenuiviriade. The strains from Guadeloupe are highlighted with red squares, strains derived from 378 Belgian Aag2 cells with black diamonds, strains from Kenya with brown square, and from Puerto Rico with blue 379 square. Representative Orthonairovirus strains belonging to the Nairoviridae are used as outgroup. 


\subsubsection{Phylogeny of Guadeloupe mosquito virus related viruses}

382 Twelve viral genomes with complete coding regions and similar to GMV, Wenzhou sobemolike virus 4 and Hubei mosquito virus 2 were obtained from all the analyzed virome datasets, including six pools of field Ae. albopictus from China (17-GZ-larvae, 17-GZ-adult, 18-GZlarva, 18-GZ-pupa, 18-GZ-adult, SRR7204303-CN-2016), three pools of Ae. aegypti and Cx. quinquefasciatus mixture from Puerto Rico (SRR3168916-PR-2014, SRR3168921-PR-2014, SRR3168924-PR-2014), two Ae. aegypti pools from Kenya (18-Kenya1 and 18-Kenya2) and one from Thailand (SRR6155879-TH-2015). The viral genomes contain two segments, encoding an RdRp and one hypothetical protein on segment 1 (1500 - $1600 \mathrm{nt})$, and a capsid and one hypothetical protein encoded by segment 2 (3000 - $3200 \mathrm{nt})$. GMV, Wenzhou sobemo-like virus 4 and Hubei mosquito virus 2 are all newly described and currently unclassified viruses with distant relationship to the family Luteoviridae and Sobemoviridae [56].

The phylogenetic analysis based on segment 1 and 2 indicated that these sequences could be separated into three clades (Fig. 6). The genomes identified from Thailand in 2015, Puerto Rico in 2014 and 1 strain from Kenya clustered together with the Guadeloupe mosquito viruses found in Guadeloupe in 2016 and 2017 to form clade-1, with the nucleotide identity within this clade ranging from $90 \%-99 \%$ for both segments. The genomes recovered from Ae. albopictus collected from Guangzhou in 2017 and 2018 cluster together with the one recovered from the same host of Yunnan in 2016 and Wenzhou sobemo-like virus 4 in cladeconsisted of the Hubei mosquito virus 2 and the sequences from 2018-Kenya2, with nucleotide identities ranging from $70 \%-100 \%$. The nucleotide similarities for both segments among these three clades ranged from $54 \%$ to $69 \%$. 

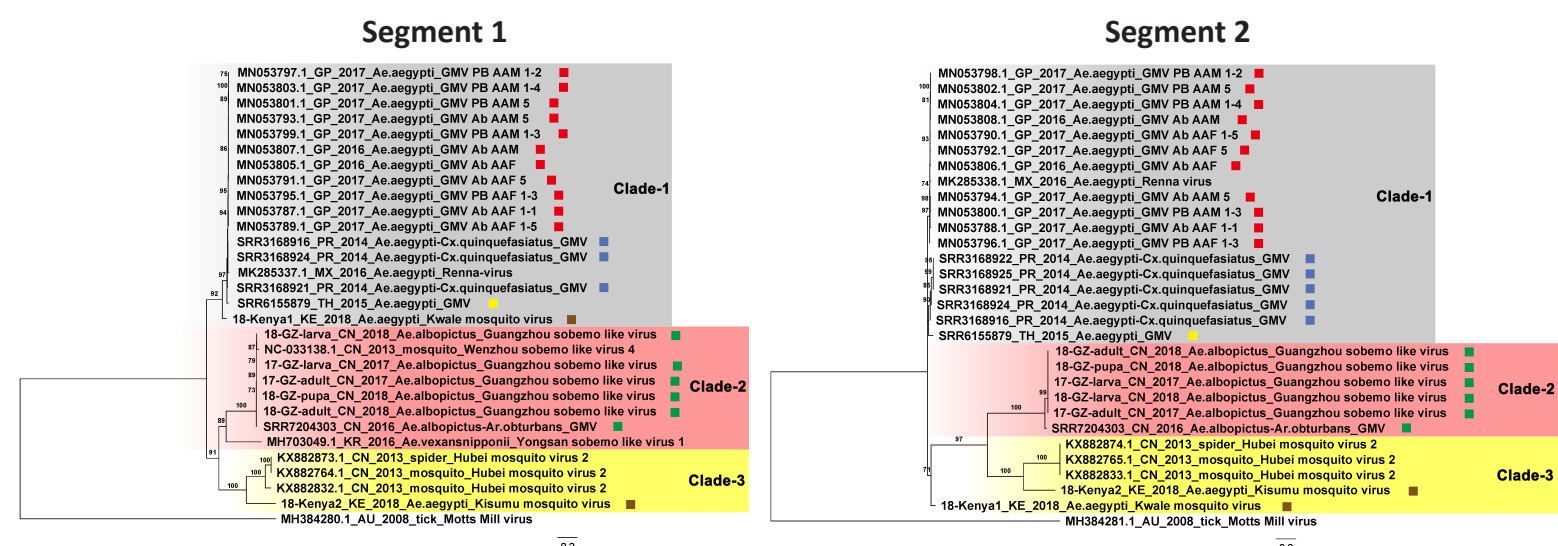

Fig. 6 Maximum-likelihood phylogenetic tree based on nucleotide sequence of the complete coding regions in segment 1 and 2 of Guadeloupe mosquito virus related viruses. The red, blue, yellow, brown and green squares after the sequence names highlight the strains from Guadeloupe, Puerto Rico, Thailand, Kenya and China, respectively. Motts Mill virus identified from tick is used as the outgroup.

\section{Discussion}

In this study, we firstly characterized the eukaryotic virome across different developmental stages of lab-derived and field collected Ae. albopictus from China. As could be expected, the virome diversity in lab Ae. albopictus is lower than that in field mosquitoes (Fig. 2), probably due to the less complex environment and clean water and food resources. Unlike bacterial communities in mosquitoes [57-62], it is interesting to note that the virome in lab-derived Ae. albopictus is very stable across all stages, consistent with a vertical transmission of these viruses. Only for the larvae, relatively few viral reads were identified. This finding suggests that the virus might remain dormant at a very low concentration without or with very low rates of replication. The fact that these viruses are also present in field mosquitoes suggest that Ae. albopictus contains a "vertically transmitted core virome" as was described before for Ae. aegypti and Culex quinquefasciatus from Guadeloupe [56]. In addition, another set of viruses was identified to be shared by the field collected Ae. albopictus mosquitoes across different stages, suggesting that they have a richer "core virome". Whether or not these additional viruses were acquired from the environment, or that the lab derived mosquitoes lost these viruses in captivity, remains to be determined. All these vertically transmitted core viromes in Ae. albopictus deserve more attention with respect to their effects on vector 
competence for important medically relevant arboviruses. The larva pool collected in the wild in 2017 is an outlier, as it contained a lot of unique viruses, which probably originated from the particular water environment they lived in, which could be contaminated by other coinhabiting insects or larvae. Alternatively, it could be that a number of mosquitoes, other than Ae. albopictus were included in the pool by accident. These results further suggest a significant influence of the breeding site ecology on the mosquito viral community.

Since some viruses (e.g. PCLPV, GMV and Aedes aegypti totivirus) identified in Ae. albopictus from China were also identified as part of the core virome in Ae. aegypti from Guadeloupe [33], we were interested to find out how these core viruses in Aedes mosquitoes were further distributed around the world. Therefore, we explored 46 viral metagenomic datasets of Aedes sp. from public database (SRA) and two of Ae. aegypti from Kenya. Although the samples are from different countries in different continents (China, US, Australia, Kenya, Thailand, Puerto Rico, Guadeloupe), from different mosquito species (Ae. aegypti, Ae. albopictus, Ae. alboannulatus, Ae. camptorhynchus), were treated using different wet-lab and sequencing procedures, used different amounts of pooled mosquitoes and sequencing depths, a highly prevalent set of widely distributed viruses could be identified on species or family level, such as PCLPV, GMV and Totiviridae (Fig. 3). How these conserved viruses in Aedes mosquitoes interact with an arbovirus infection are interesting points for further studies. A previous study explored the effect of PCLPV together with Cell-fusing agent virus (CFAV) on the replication of arboviruses in cell line Aa23 derived from Ae. albopictus [63]. The Aa23 cell lines persistently infected with CFAV was further inoculated with PCLPV. CFAV-PCLPV positive Aa23 cells strongly inhibit the growth of two flaviviruses (ZIKV and DENV) and completely blocked the infection of bunyavirus La suggest that the data generated from laboratory cell lines persistently infected by mosquito- 
specific viruses (MSVs) should be interpreted with caution. On the other hand, the intra-

MSVs interaction need to be considered when the influence of MSVs on vector competence is explored.

Although many viruses are very prevalent in Aedes mosquitoes, such as Chuvirus Mos8Chu0,

Kaiowa virus, Wuhan mosquito virus 6, Whidbey virus, Aedes aegypti totivirus and

Australian Anopheles totivirus, phylogenetic analysis was further performed on GMV and

PCLPV which had the greatest number of coding complete genomes, and APV that is highly abundant in both field and lab Ae. albopictus from China. APV was distantly relates to the YBV1 identified from Ae. vexans from South Korea forming a separate clade. All the Chinese strains from both lab and field collected mosquitoes were very similar (Fig. 4). For PCLPV, the genomes found in samples from different years, locations and habitats are very similar for all three segments (Fig. 5). Interactions between bunyaviruses and arthropods has been demonstrated over 20 million years [64-68]. The findings that both APV and PCLPV seems to be very closely related is puzzling and might suggest a rather relatively recent spread of this virus, or a very slow evolutionary rate, which might be unexpected for RNA viruses. However, the effects of these viruses on their host is very poorly understood. A recent study revealed that Aag2 cells with pre-existing infection with PCLPV do not affect the infection and growth of the mosquito-borne viruses in genus Flavivirus, Alphavirus and Rhabdovirus in cell culture [69]. GMV is a recently described two-segmented, currently unclassified virus. Three separate clades are observed for GMVs and GMV-related viruses, largely clustering according to locations. This group of viruses should be proposed as a new family and their role in arboviruses infection need to be further studied.

474 In summary, our results reveal that the virome is stable across different stages in both lab and

475 field Aedes albopictus, and that a number of core viruses exists in Aedes mosquitoes of at 476 least four species from seven countries. Since the number of the available Aedes virome data 
477 sets is still rather limited, and wet-lab procedures, sequencing depths and pool size differ

478 strongly between the analyzed data sets, the core viruses need to be further confirmed by

479 NGS or PCR. To fully characterize and understand the genetic and phenotypic diversity of 480 mosquito-specific viruses, samples from individual Aedes mosquitoes from more locations, species and time points, processed and sequenced with the same method should be analyzed.

\section{Funding}

This work was supported by the Ministry of Science and Technology of the People's Republic of China [2018ZX10101004]; the National Health Commission of the People's Republic of China [2018ZX10711001-006]; the Chinese Academy of Sciences [153211KYSB20160001]; the Wuhan

\section{Competing interests}

The authors declare that they have no competing interests.

\section{$492 \quad$ Additional files}

493 Additional file 1: SRA datasets used in this study

Additional file 2: Accession number of YBV1, PCLPV and GMV related viruses identified in this study.

496 Additional file 3: Reads number of each eukaryotic viral species in each sequencing sample

497 Additional file 4: Coverage of each eukaryotic viral species in each sequencing sample

Additional file 5: Alpha diversity of virome in field and lab Aedes albopictus 


\section{Reference}

502 1. Paupy, C., et al., Aedes albopictus, an arbovirus vector: From the darkness to the light. 
25. Xia, H., et al., Comparative Metagenomic Profiling of Viromes Associated with Four Common Mosquito Species in China. Virol Sin, 2018. 33(1): p. 59-66.

26. Shi, C., et al., A metagenomic survey of viral abundance and diversity in mosquitoes from Hubei province. PLoS One, 2015. 10(6): p. e0129845.

27. Atoni, E., et al., Metagenomic Virome Analysis of Culex Mosquitoes from Kenya and China. Viruses, 2018. 10(1).

28. Sadeghi, M., et al., Virome of $>12$ thousand Culex mosquitoes from throughout California. Virology, 2018. 523: p. 74-88.

29. Fauver, J.R., et al., West African Anopheles gambiae mosquitoes harbor a taxonomically diverse virome including new insect-specific flaviviruses, mononegaviruses, and totiviruses. Virology, 2016. 498: p. 288-299.

30. Hameed, M., et al., A viral metagenomic analysis reveals rich viral abundance and diversity in mosquitoes from pig farms. Transbound Emerg Dis, 2019.

31. Ohlund, P., et al., Viromics Reveal a Number of Novel RNA Viruses in Swedish Mosquitoes. Viruses, 2019. 11(11).

32. Pettersson, J.H., et al., Meta-Transcriptomic Comparison of the RNA Viromes of the Mosquito Vectors Culex pipiens and Culex torrentium in Northern Europe. Viruses, 2019. 11(11).

33. Shi, C., et al., Stable distinct core eukaryotic viromes in different mosquito species from Guadeloupe, using single mosquito viral metagenomics. Microbiome, 2019. 7(1): p. 121.

34. Bolger, A.M., M. Lohse, and B. Usadel, Trimmomatic: a flexible trimmer for Illumina sequence data. Bioinformatics, 2014. 30(15): p. 2114-20.

35. Bankevich, A., et al., SPAdes: a new genome assembly algorithm and its applications to single-cell sequencing. J Comput Biol, 2012. 19(5): p. 455-77.

36. https://bitbucket.org/MAVERICLab/docker-clustergenomes.

37. Buchfink, B., C. Xie, and D.H. Huson, Fast and sensitive protein alignment using DIAMOND. Nat Methods, 2015. 12(1): p. 59-60.

38. Ondov, B.D., N.H. Bergman, and A.M. Phillippy, Interactive metagenomic visualization in a Web browser. BMC Bioinformatics, 2011. 12: p. 385.

39. https://github.com/BiolnfoTools/BBMap.

40. Gu, Z., R. Eils, and M. Schlesner, Complex heatmaps reveal patterns and correlations in multidimensional genomic data. Bioinformatics, 2016. 32(18): p. 2847-9.

41. Wickham, H., ggplot2: Elegant Graphics for Data Analysis. Springer-Verlag New York, 2016.

42. McMurdie, P.J. and S. Holmes, phyloseq: an R package for reproducible interactive analysis and graphics of microbiome census data. PLoS One, 2013. 8(4): p. e61217.

43. Shi, M., et al., High-Resolution Metatranscriptomics Reveals the Ecological Dynamics of Mosquito-Associated RNA Viruses in Western Australia. J Virol, 2017. 91(17).

44. Zakrzewski, M., et al., Mapping the virome in wild-caught Aedes aegypti from Cairns and Bangkok. Scientific Reports, 2018. 8.

45. Xiao, P.P., et al., Metagenomic Analysis of Flaviviridae in Mosquito Viromes Isolated From Yunnan Province in China Reveals Genes From Dengue and Zika Viruses. Frontiers in Cellular and Infection Microbiology, 2018. 8.

46. Souvorov, A., R. Agarwala, and D.J. Lipman, SKESA: strategic k-mer extension for scrupulous assemblies. Genome Biology, 2018. 19.

47. Li, H. and R. Durbin, Fast and accurate short read alignment with Burrows-Wheeler transform. Bioinformatics, 2009. 25(14): p. 1754-60.

48. Li, H., et al., The Sequence Alignment/Map format and SAMtools. Bioinformatics, 2009. 25(16): p. 2078-9.

49. Katoh, K., et al., MAFFT: a novel method for rapid multiple sequence alignment based on fast Fourier transform. Nucleic Acids Res, 2002. 30(14): p. 3059-66. 
50. Capella-Gutierrez, S., J.M. Silla-Martinez, and T. Gabaldon, trimAl: a tool for automated alignment trimming in large-scale phylogenetic analyses. Bioinformatics, 2009. 25(15): p. 1972-3.

51. Nguyen, L.T., et al., IQ-TREE: a fast and effective stochastic algorithm for estimating maximum-likelihood phylogenies. Mol Biol Evol, 2015. 32(1): p. 268-74.

52. Kalyaanamoorthy, S., et al., ModelFinder: fast model selection for accurate phylogenetic estimates. Nat Methods, 2017. 14(6): p. 587-589.

53. https://github.com/rambaut/figtree/releases.

54. Sanborn, M.A., et al., Metagenomic Analysis Reveals Three Novel and Prevalent Mosquito Viruses from a Single Pool of Aedes vexans nipponii Collected in the Republic of Korea. Viruses, 2019. 11(3).

55. Zhang, X., et al., Discovery and high prevalence of Phasi Charoen-like virus in field-captured Aedes aegypti in South China. Virology, 2018. 523: p. 35-40.

56. Shi, C., et al., Stable distinct core eukaryotic viromes in different mosquito species from Guadeloupe, using single mosquito viral metagenomics. Microbiome, 2019.

57. Chavshin, A.R., et al., Malpighian tubules are important determinants of Pseudomonas transstadial transmission and longtime persistence in Anopheles stephensi. Parasit Vectors, 2015. 8: p. 36.

58. Coon, K.L., M.R. Brown, and M.R. Strand, Gut bacteria differentially affect egg production in the anautogenous mosquito Aedes aegypti and facultatively autogenous mosquito Aedes atropalpus (Diptera: Culicidae). Parasit Vectors, 2016. 9(1): p. 375.

59. Favia, G., et al., Bacteria of the genus Asaia stably associate with Anopheles stephensi, an Asian malarial mosquito vector. Proc Natl Acad Sci U S A, 2007. 104(21): p. 9047-51.

60. Chen, S., M. Bagdasarian, and E.D. Walker, Elizabethkingia anophelis: molecular manipulation and interactions with mosquito hosts. Appl Environ Microbiol, 2015. 81(6): p. 2233-43.

61. Coon, K.L., et al., Mosquitoes rely on their gut microbiota for development. Molecular Ecology, 2014. 23(11): p. 2727-2739.

62. Moll, R.M., et al., Meconial peritrophic membranes and the fate of midgut bacteria during mosquito (Diptera: Culicidae) metamorphosis. J Med Entomol, 2001. 38(1): p. 29-32.

63. Schultz, M.J., H.M. Frydman, and J.H. Connor, Dual Insect specific virus infection limits Arbovirus replication in Aedes mosquito cells. Virology, 2018. 518: p. 406-413.

64. Ballinger, M.J., et al., Discovery and Evolution of Bunyavirids in Arctic Phantom Midges and Ancient Bunyavirid-Like Sequences in Insect Genomes. Journal of Virology, 2014. 88(16): p. 8783-8794.

65. Li, C.X., et al., Unprecedented genomic diversity of RNA viruses in arthropods reveals the ancestry of negative-sense RNA viruses. Elife, 2015. 4.

66. Qin, X.C., et al., A tick-borne segmented RNA virus contains genome segments derived from unsegmented viral ancestors. Proceedings of the National Academy of Sciences of the United States of America, 2014. 111(18): p. 6744-6749.

67. Tokarz, R., et al., Genome characterization of Long Island tick rhabdovirus, a new virus identified in Amblyomma americanum ticks. Virology Journal, 2014. 11.

68. Tokarz, R., et al., Virome Analysis of Amblyomma americanum, Dermacentor variabilis, and Ixodes scapularis Ticks Reveals Novel Highly Divergent Vertebrate and Invertebrate Viruses. Journal of Virology, 2014. 88(19): p. 11480-11492.

69. Fredericks, A.C., et al., Aedes aegypti (Aag2)-derived clonal mosquito cell lines reveal the effects of pre-existing persistent infection with the insect-specific bunyavirus Phasi Charoenlike virus on arbovirus replication. PLoS Negl Trop Dis, 2019. 13(11): p. e0007346. 